\title{
A programmable tone generator for presenting melodic and harmonic patterns in music research
}

\author{
DAVID BRIAN WILLIAMS and RICHARD K. HOSKIN \\ SWRL Educational Research and Development, 4665 Lampson Avenue, Los Alamitos, Califomia 90720
}

\begin{abstract}
This report describes the design of a programmable tone generator, a digitally controlled system for generating complex tones. The PTG will provide sequential series of single tones or simultaneously sounding two- and three-tone groups over a four-octave range. Programming of the PTG is accomplished by paper tape using a digital, binary configuration. The current version will interface with Grason-Stadler Series 1200 equipment.
\end{abstract}

The programmable tone generator described herein was developed for a music research laboratory and is used to generate sequences of melodic and harmonic patterns for experiments in children's music perception. The laboratory is installed in a trailer, transportable to school settings. Timing, sequencing, and logic control of audio and visual instrumentation are effected by Grason-Stadler, Series 1200 equipment.

The programmable tone generator (PTG) is a digitally controlled sound-generating system capable of producing sequential series of single tones or simultaneously sounding two- and three-tone groups. The instrument has a four-octave range $(65.4$ to $987.8 \mathrm{~Hz})$ set to equaltempered tuning and can produce either a square or a triangle waveform, or some combination thereof.

The instrument allows an adequate number of sequential tone groups to be programmed via paper tape (e.g., 500 six-tone patterns). Tone groups, or PTG word units, are programmed on paper tape in a particular binary format. The punched tape is then mounted onto the transport-drive system of the PTG. One-inch fan-folded black paper tape is required. When PTG is triggered by a 1-logic signal from any of the GrasonStadler modules, the binary codes in one PTG frame are read through a photocell sensing unit and the tone group sounds. Upon termination of the 1-logic signal, the drive system advances to the next frame.

\section{PROGRAMMING}

The structure of a PTG frame consists of seven punched rows, eight columns wide (Figure 1). Rows A, $\mathrm{B}$, and $\mathrm{C}$ are reserved for coding from one to three tone requests (these tones sound simultaneously). The tone selection is made from decimal numbers 1 to 12 , a chromatic octave, and is punched in columns 1 through 4

The work upon which this publication is based was performed pursuant to Contract NE-C-00-3-0064 with the National Institute of Education, Department of Health, Education, and Welfare. Correspondence concerning the article should be sent to David Brian Williams, Department of Music, Illinois State University, Normal, Illinois 61761 . as 4-bit binary. Rows a, b, and c are reserved for coding the octave requests (from the first to the fourth octave or from 65.4 to $987.8 \mathrm{~Hz}$ ) for each respective tone in the PTG word. Each PTG frame is terminated with an 8-bit end-of-word character, binary 01111100.

As an additional feature, the binary coding of each PTG frame on the paper tape appears as 1-logic $(-10-\mathrm{V} \mathrm{dc})$ signals at a 3 by 4 matrix of output pin jacks on the front panel of the instrument. If all three channels are not used for tone generation, then this output offers the option of using either the $A, B$, or $C$ channel to program control over Grason-Stadler modules simultaneously with the tone coding, e.g., to trigger a timer or programmable attenuator. The binary coding procedure for the PTG frame structure is basic enough to make hand punching of the paper tape possible. However, a FORTRAN program has been designed for a PDP-computer system to prepare the programmed tape (Williams, Note 1).

\section{CONSTRUCTION}

Figure 2 shows the electronic circuit for one of the three tone channels with its respective octave selection channel. An 18-photocell cluster is used to read a PTG frame. Phototransistors $1 / 5$ in. in diam were used. Tapered acrylic light guides were cast on the viewing end of each photocell and then slanted into position for mounting on $1 / 10$-in. centers to a standard perforated circuit board. The outside of each light guide/phototransistor was sprayed with dull black lacquer to insure absence of crosstalk. Black 1-in. paper tape was used to achieve the greatest opacity.

The translation of the binary tone coding into discrete positions in the musical octave is achieved by an integrated circuit N74154. The mutually exclusive outputs are amplified by 7414 hex buffers driving reed relays that connect in the appropriate resistor to the 566 function generator.

To effect selection among the four octaves, three relays per channel alter the voltage-controlled input to the 566 function generator. Each octave has a 20-turn 


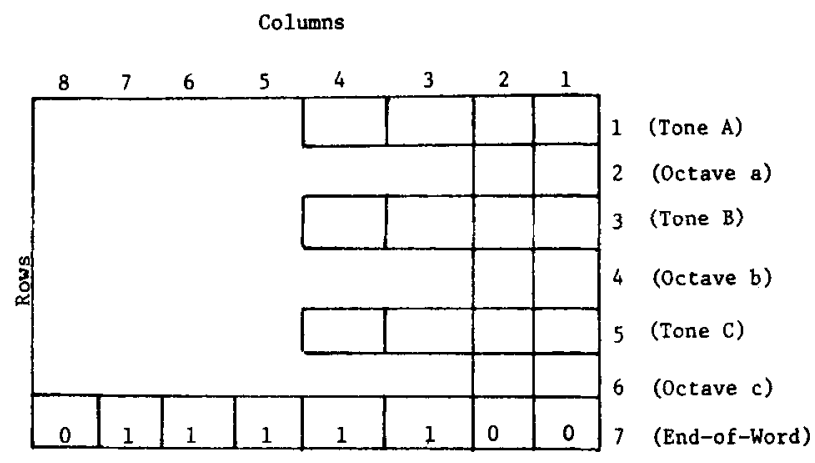

Figure 1. Structure of a PTG frame.

potentiometer to set the bottom note in the octave, with the other tones following in the ratios set by 12 individual frequency control potentiometers. Octave tuning proceeds from the lowest octave. Some nonlinearity in the function generator requires compromise in adjustment to keep the overall tolerances within acceptable limits. Tuning is accurate within $1 \%$.

In order to advance seven rows of the paper tape at a time (one PTG frame), a lightweight forked arm moves up to engage the five holes punched in the last row of a frame. The arm is then driven horizontally until the next frame has been moved into the reading position over the photocell cluster. After the arm has advanced, it moves down and around into the stop position. A small dc motor drives the arm through a single pulley reduction. The connection between the drive crank pin and the moving arm is by means of an extension spring that gives an accelerated motion at certain parts of the revolution. Minimum advance time with 1 -logic signals as a trigger is $150 \mathrm{msec}$.

Power to the integrated circuits and reed relays is provided by a $5 . \mathrm{V}$ regulated power supply using an LM 309K integrated cricuit regulator. Another regulated supply gives $12 \mathrm{~V}$ for the three function generators.

The $-10-\mathrm{V} d c$ input signal is amplified by a single power transistor that operates a relay and lamp circuit. As long as the input signal persists, the oscillator outputs are connected to the output jacks by reed relays. In this way, frequency duration is defined by the duration of external timing control. In addition, a capacitor is connected to a charging circuit. As soon as the input signal decays, the outputs are disconnected and the charged-up capacitor triggers an SCR that starts the advance motor. The motor advances one PTG frame, then triggers the SCR to the off position. With this system, any noise generated by the tape advance is isolated from the output circuits.

The three function generators have both squarewave and triangle-wave outputs. The two types of out puts are fed into a timbre control mixing circuit that

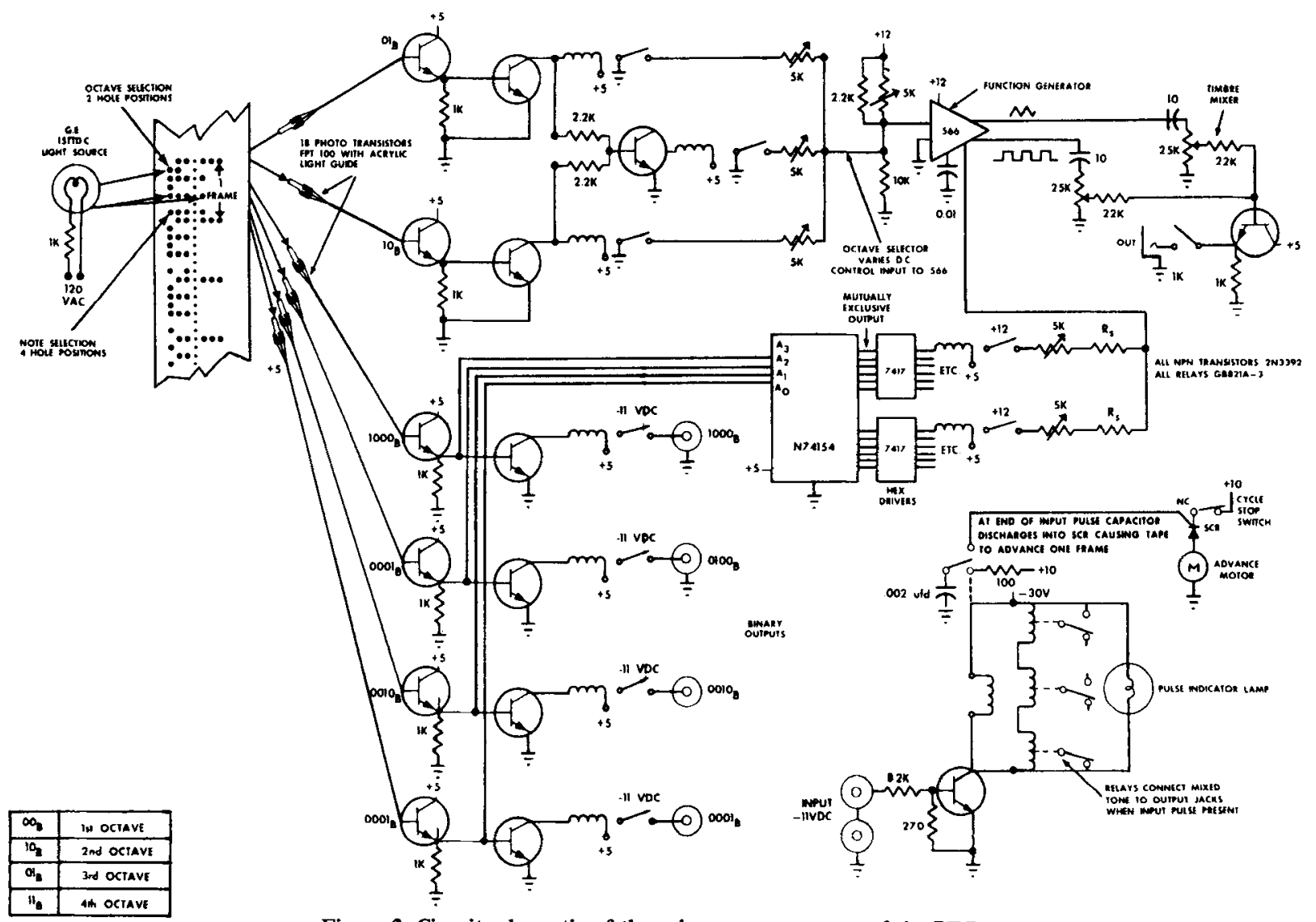

Figure 2. Circuit schematic of the primary components of the PTG. 
allows any ratio of the two waveforms to be established. Emitter coupled output amplifiers isolate the mixers from effects of line loading.

A 1/4-W monitor amplifier is included on the panel to facilitate testing and adjustment of timbre and tone parameters. A local test switch is located on the front panel near the input. An indicator lamp is activated by the input signal. All components are mounted on either circuit boards, steel chassis, or the front panel.

Frequency stability of the tone generators is within $.5 \%$ over the temperature ranges encountered in the test location. Temperatures in the research trailer range from $4^{\circ} \mathrm{C}$ during the night to $27^{\circ} \mathrm{C}$ during the day. The principal effect of temperature variation is on the activity of the phototransistors used in the tape sensing unit. Their performance is stabilized by providing a 15 -min warm-up period before operation. A cooling fan added to the regulated power supply improves circulation of air across the heat exchanger.

\section{REFERENCE NOTE}

1. Williams, D. B. PTGBP: A FORTRAN program for generating binary punched paper tapes for the PTG. (Technical Note TN 2-74-13.) SWRL Educational Research and Development. Los Alamitos. California, 1974.

(Received for publication May 24, 1976.) 